\title{
Accurate EPMA Quantification of the First Series Transition Metals using Ll Lines
}

\author{
M. Moret, C. Hombourger, and M. Outrequin \\ CAMECA, 29 Quai des Grésillons, 92622 Gennevilliers Cedex, France \\ mona.moret@ametek.com
}

In conventional EPMA, X-ray $\mathrm{K}$ lines are used for accurate quantification of the transition metal elements. However, at low beam accelerating voltages (i.e., $<5 \mathrm{keV}$ ), only low-energy $\mathrm{X}$-ray lines are emitted, including $\mathrm{K}$ lines for $\mathrm{Z} \leq 22$ and $\mathrm{L}$ and $\mathrm{M}$ lines for $\mathrm{Z}>22$. Low beam energy operation offers several advantages: improvement of analytical spatial resolution and reduction of both secondary fluorescence and sample charging effects. The use of lowenergy X-ray lines for quantitative analysis does present new analytical challenges, though, because these lines are subject to larger peak shifts, more line overlaps and lower fluorescence yields, as compared to higher-energy $\mathrm{K}$ lines. The low yields also reduce the intensity of certain lines, as does the low overvoltage, $U$ (defined as the ratio of beam energy to ionization energy for a given line), which lowers the ionization probability for the X-ray line.

If we consider the example of $\mathrm{Fe}(\mathrm{Z}=26)$ analyzed at $15 \mathrm{keV}$, many characteristic $\mathrm{X}$-ray lines (K- and L-series) are produced from the atom. The X-ray lines traditionally used for quantification are the $\mathrm{K} \alpha$ line (transition from $\mathrm{L}_{3}$ sub-shell to $\mathrm{K}$ sub-shell) and $\mathrm{L} \alpha$ line (transition from $\mathrm{M}_{5}$ sub-shell to $\mathrm{L}_{3}$ sub-shell). Generation of the Fe $\mathrm{L} \alpha$ line involves valence electrons, which are affected by the chemical bonding of Fe in the target sample. Wavelength shifts, peak shape modifications, and increases or decreases in the relative intensities of the characteristic lines can be readily observed between different chemical types.

In general, for elements of the first transition metal series (Sc to $\mathrm{Zn}$ ), the pattern of $\mathrm{L}$ emission spectra varies according to the energy of the incident electrons $\left(E_{0}\right)$, as follows:

- $\mathrm{E}_{0}$ between the $\mathrm{L}_{3}$ and $\mathrm{L}_{2}$ threshold energies: intensity of the high energy satellite lines is reduced.

- $\mathrm{E}_{0}$ from the $\mathrm{L}_{3}$ sub-shell threshold energy up to 3 times this energy: excitation and development of the high energy satellites increases and the shape of the $L \alpha$ line becomes increasingly distorted.

- $\quad E_{0}$ above 3 and 4 times the $L_{3}$ sub-shell threshold energy: the absorption path of the generated $\mathrm{X}$-rays increases and thus the fine structure on the high energy side "disappears" due to selfabsorption.

From these spectroscopic observations, it becomes obvious that the shape and the peak position of the $\mathrm{L}$ emission band is strongly dependent on the incident electron energy and thus can lead to inaccuracies in quantification when using $L \alpha$ lines.

As an alternative, Fialin et al. [1] have suggested the use of the Ll line, instead of the more commonly used L $\alpha$ or L $\beta$ lines. The Ll line has the advantage of being independent of the chemical state of the element, since the electrons of the outer shell involved in the Ll transition are core, not valence, electrons. One drawback is the low intensity of Ll, compared to the L $\alpha$ line (typically 10 times less for Fe measured with a TAP crystal). Quantitative results achieved by using the $\mathrm{Ll}$ lines for the elements of the first transition series will be presented. 
[ 1] Fialin M, Wagner C and Remond G, 1998 in: Proceedings, EMAS’98, 3rd Regional Workshop.

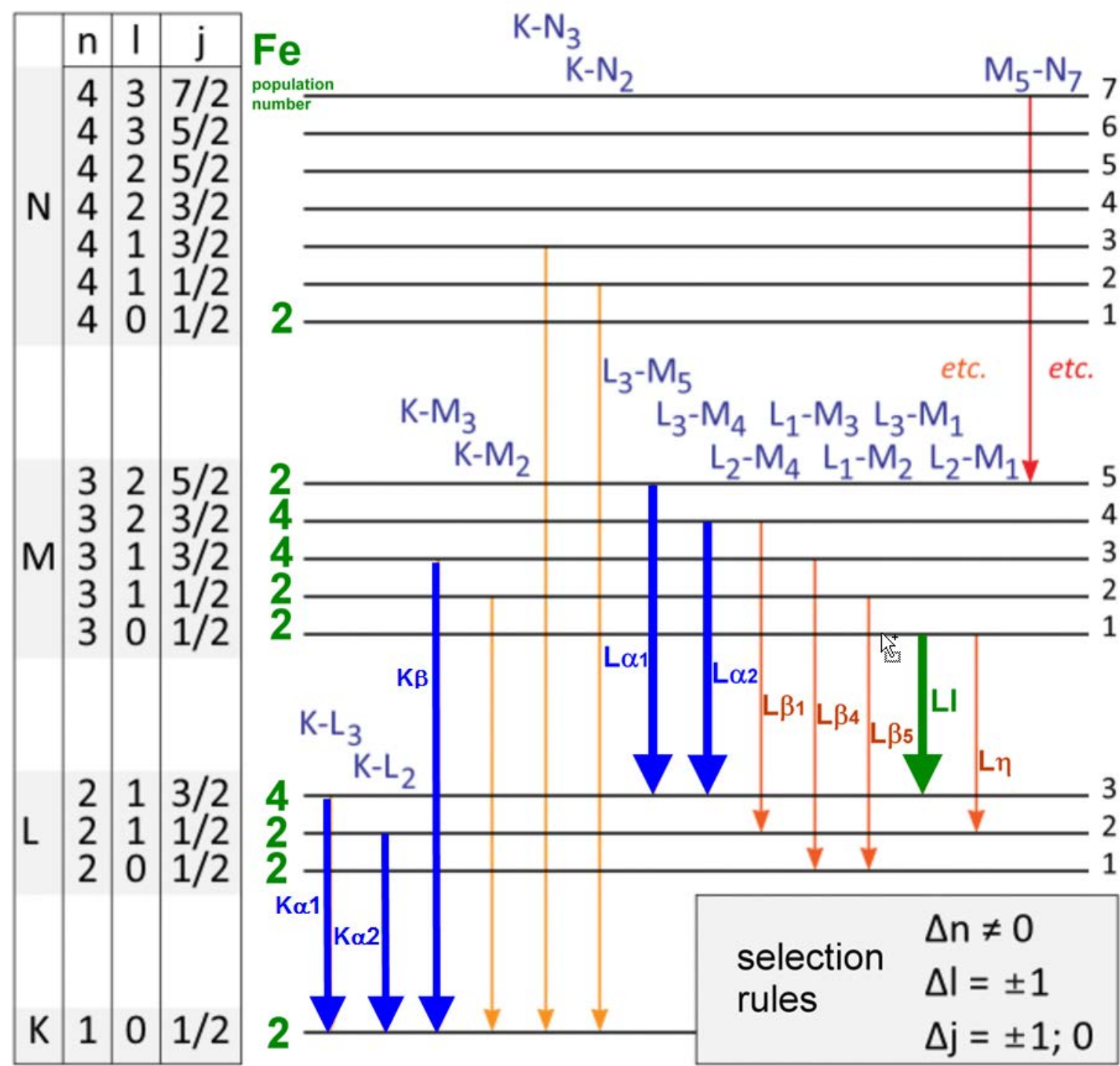

Figure 1: Energy level diagram showing electron transitions producing Fe $\mathrm{K}$ and L X-rays. 